\title{
Effects of solvent evaporation on water sorption/ solubility and nanoleakage of adhesive systems
} Talita Baumgratz Cachapuz CHIMELI'1, Paulo Henrique Perlatti D’ALPINO², Patrícia Nóbrega PEREIRA ${ }^{1}$, Leandro
Augusto HILGERT ${ }^{1}$, Vinicius DI HIPÓLITO², Fernanda Cristina Pimentel GARCIA ${ }^{1}$

1- Department of Operative Dentistry, School of Dentistry, University of Brasilia-UnB, Brasília, DF, Brazil.
2- Biomaterials Research Group, School of Dentistry, Anhanguera University of São Paulo (UNIAN-SP), São Paulo, SP, Brazil.

Corresponding address: Fernanda C. P. Garcia - Universidade de Brasília - UNB QRSW 05 - Bloco B3 - Apto 302 - Sudoeste - Brasília - DF - Brazil - 70675523 Phone : 556181127078 - Fax: 556133721055 - e-mail: garciafcp@unb.br

Submitted: November 22, 2013 - Modification: March 26, 2014 - Accepted: April 6, 2014

\section{ABSTRACT}

\begin{abstract}
$\mathrm{O}$ bjective: To evaluate the influence of solvent evaporation in the kinetics of water diffusion (water sorption-WS, solubility-SL, and net water uptake) and nanoleakage of adhesive systems. Material and Methods: Disk-shaped specimens $(5.0 \mathrm{~mm}$ in diameter $\mathrm{x}$ $0.8 \mathrm{~mm}$ in thickness) were produced $(\mathrm{N}=48)$ using the adhesives: Clearfil $\mathrm{S}^{3}$ Bond (CS3)/ Kuraray, Clearfil SE Bond - control group (CSE)/Kuraray, Optibond Solo Plus (OS)/Kerr and Scotchbond Universal Adhesive (SBU)/3M ESPE. The solvents were either evaporated for $30 \mathrm{~s}$ or not evaporated ( $\mathrm{N}=24 /$ per group), and then photoactivated for $80 \mathrm{~s}$ ( $550 \mathrm{~mW} /$ $\left.\mathrm{cm}^{2}\right)$. After desiccation, the specimens were weighed and stored in distilled water $(\mathrm{N}=12)$ or mineral oil $(\mathrm{N}=12)$ to evaluate the water diffusion over a 7-day period. Net water uptake (\%) was also calculated as the sum of WS and SL. Data were submitted to 3-way ANOVA/ Tukey's test $(\alpha=5 \%)$. The nanoleakage expression in three additional specimens per group was also evaluated after ammoniacal silver impregnation after 7 days of water storage under SEM. Results: Statistical analysis revealed that only the factor "adhesive" was significant $(p<0.05)$. Solvent evaporation had no influence in the WS and SL of the adhesives. CSE (control) presented significantly lower net uptake (5.4\%). The nanoleakage was enhanced by the presence of solvent in the adhesives. Conclusions: Although the evaporation has no effect in the kinetics of water diffusion, the nanoleakage expression of the adhesives tested increases when the solvents are not evaporated.
\end{abstract}

Keywords: Dentin-bonding agents. Solvents. Solubility. Leakage.

\section{INTRODUCTION}

Current restorative techniques are associated with the bonding characteristics of resin-based materials. Contemporary dentin bonding agents contain a chemically balanced combination of hydrophilic (i.e. HEMA, BPDM, PENTA) and hydrophobic monomers (i.e. Bis-GMA, UDMA) that intrinsically permeate the wet dentin surface ${ }^{24}$. In addition, the dentin bonding agents contain solvents, known to be essential components for establishing adequate dentin-resin interface. Solvents are responsible for carrying these resin monomers throughout the collagen fibrils of the dentin substrate after the etching step ${ }^{17,19}$. In these dental adhesives, higher concentrations of relatively hydrophilic monomers are used. This characteristic complex blend of hydrophilic/ hydrophobic ingredients, water and solvents makes the adhesives prone to phase separation, which may impair their bonding effectiveness ${ }^{26}$. In order to allow the mixing of these kinds of monomers, and also to avoid the phase separation between them, volatile organic solvents, such as ethanol and acetone, are added in the formulation of adhesives ${ }^{27}$. These solvents facilitate the evaporation of water from the wet dentin, ensuring an increased monomer infiltration into the demineralized dentin, thus improving the physical-chemical interaction with the tooth substrate ${ }^{3}$.

The solvent evaporation before the photoactivation of an adhesive system is regarded 
as being of paramount importance to increase the effectiveness of the bonding procedure ${ }^{6,9}$. The adhesive system is placed and exposed while it is in a liquid state and is relatively low in viscosity. This lowered viscosity may lead to enhanced radical mobility, and thus higher conversion values ${ }^{4}$. For a durable, long-term sealing of dentin, monomers must be converted to stable high-molecular-weight polymers ${ }^{20}$. The residual water or organic solvents is claimed to be responsible for producing localized areas of incomplete monomer polymerization ${ }^{6,7,9}$. Thus, the monomer conversion and the extent of polymerization might vary throughout the hybrid layer, which could cause significant differences in the quality of the interpenetrating network at different locations ${ }^{13}$. A direct correlation between the extent of polymerization of the adhesive films and their permeability has been found ${ }^{2}$. In addition, water is responsible for the chemical decomposition, such as oxidation and hydrolysis of the resin matrix $^{5}$. However, the water uptake is not only dependent upon the presence of residual solvent, but is also dependent upon the hydrophilicity of these materials ${ }^{28}$.

Therefore, the aim of the present study was to evaluate the influence of solvent evaporation on the water sorption, solubility, and net water uptake (sum of water sorption and solubility) of specimens immersed in water and mineral oil, and also on the nanoleakage pattern of contemporary adhesive systems. The hypotheses tested were: (I) the solvent evaporation would reduce the net water uptake; (II) the water sorption and solubility parameters will be similar, irrespective of the

\begin{tabular}{|c|c|c|c|}
\hline Adhesives & Composition & Manufacturer & Batch \# \\
\hline Clearfil SE Bond (CSE) & $\begin{array}{c}\text { Primer: MDP, HEMA, } \\
\text { Dimethacrylates, } \\
\text { Di-canforquinone, } \\
\text { N,N-Dietanol-p-toluidina, } \\
\text { Water. } \\
\text { Bond: MDP, BisGMA, HEMA, } \\
\text { Dimethacrylates, } \\
\text { Di-canforquinone } \\
\text { N,N-Diethanol-p-toluidine } \\
\text { Silanized colloidal silica. }\end{array}$ & Kuraray, Osaka, Japan & $1548 \mathrm{AA}$ \\
\hline Clearfil $\mathrm{S}^{3}$ Bond (CS3) & $\begin{array}{c}\text { 10-MDP, HEMA, BisGMA, } \\
\text { Water, Ethanol, } \\
\text { Silanized colloidal silica, } \\
\text { Camphorquinone. }\end{array}$ & Kuraray, Osaka, Japan & 00156B \\
\hline Optibond Solo Plus (OP) & $\begin{array}{l}\text { Ethyl alcohol, } \\
\text { Alkyl dimethacrylate resins, } \\
\text { Barium aluminoborosilicate glass, } \\
\text { Fumed silica (silicon dioxide), } \\
\text { Sodium hexafluorosilicate. }\end{array}$ & Kerr, Orange, CA, USA & 3533713 \\
\hline Scotchbond Universal (SBU) & $\begin{array}{c}\text { BisGMA, 2-Hydroxyethyl Methacrylate, } \\
\text { Decamethylene, Dimethacrylate, Ethanol, } \\
\text { Water, } \\
\text { Silane Treated Silica, } \\
\text { 2-Propenoic Acis, 2-Methyl-, Reaction } \\
\text { products with 1, 10-Decanediol and } \\
\text { Phosphorous oxide (P2O5), Copolymer of } \\
\text { acrylic and itaconic acid, camphorquinone, } \\
\text { dimethylaminobenzoat (-4) toluene. }\end{array}$ & $\begin{array}{c}\text { 3M ESPE, St. Paul, MN, } \\
\text { USA }\end{array}$ & $\mathrm{ADH}-02$ \\
\hline
\end{tabular}

MDP: methacryloyloxydecyl dihydrogen phosphate; BisGMA: bisphenol-A glycidyldimethacrylate; HEMA:2hydroxyethylmethacrylate

Figure 1- Composition, manufacturers and batch numbers of the adhesives used 
immersion media; and (III) the nanoleakage of the adhesives tested would be reduced when the solvents are evaporated.

\section{MATERIAL AND METHODS}

\section{Experimental design}

In this in vitro study, the assessments of water sorption and solubility of adhesive specimens immersed in water and mineral oil were performed according to the factors: (1) adhesive system, at four levels: I- Clearfil S3 Bond (CS3) - a onestep, self-etching adhesive -, II- Scotchbond Universal Adhesive (SBU) - a universal adhesive -, III- Clearfil SE Bond (CSE) - a two-step, selfetching adhesive - , IV- Optibond Solo Plus (OP) - a two-step, total-etching adhesive; (2) adhesive protocol: I- evaporated, II- non-evaporated; and (3) immersion media, at two levels: I- water, and II- mineral oil. For comparative reasons, only specimens of the "bond" component of CSE were fabricated. The net water uptake, i.e., the sum of water sorption and solubility, was also calculated. The composition of the adhesive systems is described in Figure 1. In order to support the results, the nanoleakage pattern of contemporary adhesive systems was also performed.

\section{Specimen fabrication}

Forty-eight disc-shaped specimens of each material (except for CSE; $n=24$ ) were prepared for each storage condition (water and mineral oil). For the specimen fabrication, adhesive systems were dispensed into a silicon mold $(5.0 \mathrm{~mm}$ in diameter $x 0.8 \mathrm{~mm}$ in thickness). For solvated adhesives (SBU, CS3, OP), oil/water-free compressed air was gently blown for $30 \mathrm{~s}$ at a distance of $10 \mathrm{~cm}$ to facilitate the solvent evaporation (E). For the non-solvated adhesive (CSE Bond), the drying step was not performed. As an air spray is recommended to optimize solvent evaporation ${ }^{10}$, care was taken to be gently applied to avoid oxygen incorporation in the adhesive specimens. In addition, care was also taken to carefully remove all visible air bubbles entrapped in the adhesive specimens, and a polyester strip was placed over the adhesive and covered with a glass slide. The specimens were photoactivated using a QTH light (Demetron LC, $450 \mathrm{Mw}$, Demetron Research Corp., Danbury, CT, USA) for $40 \mathrm{~s}$ with a power density of $550 \mathrm{~mW} / \mathrm{cm}^{2}$. The specimens were then carefully removed from the mold and photoactivated for additional $40 \mathrm{~s}$.

\section{Water sorption and solubility test}

Water sorption and solubility tests were based on the 4049 ISO standard, with the exception of the specimen's dimensions $(5.0 \mathrm{~mm}$ in diameter 0.8 $\mathrm{mm}$ in thickness). After $24 \mathrm{~h}$, the specimens were placed in a desiccator device containing silica gel and stored at $37^{\circ} \mathrm{C}$. The specimens were repeatedly weighed on an analytical balance (AG204, MettlerToledo, Columbus, OH, USA) every 24 h, until a constant mass $\left(m_{1}\right)$ was obtained (i.e. a mass in which variation amounted to less than $0.2 \mathrm{mg}$ within any $24 \mathrm{~h}$ period) ${ }^{12}$. The thickness and diameter of the specimens were measured at three different points to the nearest $0.01 \mathrm{~mm}$ using a digital caliper, and these measurements were used to calculate the volume ( $\mathrm{V}$ ) of each specimen (in $\mathrm{mm}^{3}$ ). Specimens were then individually placed in test tubes (Eppendorf vials) containing $1.5 \mathrm{~mL}$ of distilled water $\left(\mathrm{pH} \mathrm{7.2)}\right.$ at $37^{\circ} \mathrm{C}$ for 7 days. Half of the specimens $(\mathrm{N}=12)$ were placed in sealed glass vials containing $1.5 \mathrm{ml}$ of a chemically-inert mineral oil (Nujol, liquid petrolatum, Sigma-Aldrich Co., Saint Louis, MO, USA). These specimens were tested as controls in parallel with the specimens immersed in distilled water.

The storage time interval of up to 7 days had passed, the tubes were removed from the oven and left to equilibrate at room temperature for 30 min. The specimens were then washed in running water, gently wiped with a soft absorbent paper, weighed by means of an analytical balance $\left(m_{2}\right)$, and returned to vials containing $10 \mathrm{~mL}$ of fresh distilled water or oil. Following the further 7 days of storage, the specimens were dried inside a desiccator containing fresh silica gel and weighed daily until a constant mass $\left(\mathrm{m}_{3}\right)$ was obtained (as previously described). The initial mass determined after the first desiccation process $\left(m_{1}\right)$ was used to calculate the change in mass after each fixedtime interval of the 7 days of storage. Changes in mass were plotted against the storage time in order to obtain the kinetics of water sorption for the entire period of water storage. The water sorption (WS) and solubility (SL) over 7 days of water storage ${ }^{12}$ were calculated by means of the following formulae ${ }^{21}$ :

$$
W S=\frac{m_{2}-m_{3}}{V} \quad S L=\frac{m_{1}-m_{3}}{V}
$$

where $\mathrm{m} 1$ refers to the initial dry constant mass (mg) prior to immersion in water; $\mathrm{m}^{2}$ describes the mass ( $\mathrm{mg}$ ) after water immersion at various time periods; $\mathrm{m}^{3}$ is the mass $(\mathrm{mg})$ after drying the specimens that had reached their maximum water sorption; and $\mathrm{V}$ refers to the specimen volume in $\mathrm{mm}^{3}$. Net water uptake (\%) was also calculated as the sum of water sorption and solubility. Data were analyzed by three-way ANOVA (with factors including "adhesive system", "adhesive protocol", and "immersion media") and Tukey's post-hoc test for multiple comparisons, at a preset alpha of $5 \%$. 


\section{Nanoleakage}

For the nanoleakage pattern investigation, six additional disc-shaped specimens were prepared for the solvated adhesive systems, and three discs were made for CSE. In order to investigate the influence of the solvent incorporated into adhesive systems on the nanoleakage, the specimens were stored in distilled water for 7 days and then placed in an ammoniacal silver nitrate solution for $24 \mathrm{~h}$. After that, the specimens were rinsed thoroughly in distilled water and immersed in a photo developing solution for $8 \mathrm{~h}$ under a fluorescent light to reduce silver ions into metallic silver grains ${ }^{24}$. The stained specimens were then profusely water-rinsed in tap water, mounted in aluminum stubs, dehydrated in silica gel for $2 \mathrm{~h}$, and then submitted to carbon evaporation (SCD 050, Balzer Union AG, Balzers, Lichtenstein). A qualitative analysis of the nanoleakage patterns was performed using a scanning electron microscope (SEM - PhenomWorld, Eindhoven, The Netherlands), operating in back-scattering electron mode with an accelerating voltage of $15 \mathrm{kV}$. The nanoleakage patterns were classified as follow: Type I - moderate silver uptake; or Type II - severe silver uptake.

\section{RESULTS}

Water sorption and solubility

Mass variation curves for the 12 days of immersion in water are presented in Figure 2. The results are shown in Table 1. The statistical analysis revealed that only the factor "adhesive protocol" was significant $(p=0.01)$. The solvent evaporation procedure had no effect on the water sorption and solubility of the adhesives $(p=0.21)$, irrespective of the storage media. When mass gain (i.e. water sorption) and mass loss (i.e. solubility) of adhesive disks were plotted against time, nonsolvated adhesive CSE was determined to have the lowest water sorption (Figure 2). CSE adhesive also presented significantly lower means of SL (-2.9 $\left.\mu \mathrm{g} / \mathrm{mm}^{3}\right)(p<0.05)$. When the WS and SL were compared as a function of the storage media, the means were statistically lower when the specimens were immersed in mineral oil. The net water uptake means varied from 5.4 to $24.6 \%$ among the adhesive systems (Table 1) and were determined to be significantly lower for the CSE Bond (5.4\%). All the adhesives stored in water presented a time-dependent increase in water sorption and solubility, whereas the adhesives stored in mineral oil presented lower sorption and solubility means (Figure 2). In spite of the variation in mass as a function of the time, CSE was the only adhesive to present similar weight after the desiccation process in comparison to that obtained at $\mathrm{m}_{1}$ (Figure 2).

\section{Nanoleakage}

The nanoleakage patterns of the adhesives investigated are illustrated in Figures 3 and 4 .

Table 1- Water sorption (WS), solubility (SL) and net water uptake of the adhesives tested

\begin{tabular}{|c|c|c|c|c|c|c|c|}
\hline \multirow{3}{*}{$\begin{array}{l}\text { Adhesive } \\
\text { system }\end{array}$} & \multirow{3}{*}{$\begin{array}{l}\text { Adhesive } \\
\text { protocol }\end{array}$} & \multicolumn{2}{|l|}{ wS } & \multicolumn{3}{|c|}{ SL } & \multirow{3}{*}{$\begin{array}{l}\text { Net Water } \\
\text { Uptake (\%)D }\end{array}$} \\
\hline & & Water & \multirow{2}{*}{$\frac{\text { Oil }}{\left(\mu \mathrm{g} / \mathrm{mm}^{3}\right)}$} & \multicolumn{2}{|c|}{ Water } & \multirow{2}{*}{$\frac{\text { Oil }}{\left(\mu \mathrm{g} / \mathrm{mm}^{3}\right)}$} & \\
\hline & & $\left(\mu \mathrm{g} / \mathrm{mm}^{3}\right) \quad(\%) \mathrm{C}$ & & $\left(\mu \mathrm{g} / \mathrm{mm}^{3}\right)$ & $(\%) \mathrm{C}$ & & \\
\hline CSE & $\begin{array}{c}\text { Non- } \\
\text { evaporated }\end{array}$ & $57.1 \pm 5.8^{\mathrm{cA}}(5.7)$ & $7.6 \pm 2.1^{1 \mathrm{~B}}$ & $-2.9 \pm 4.9 \mathrm{~A}$ & $(0.2)$ & $1.1 \pm 3.4^{\mathrm{aA}}$ & $5.9 \pm 0.9^{a}$ \\
\hline \multirow[t]{2}{*}{$\operatorname{cs} 3$} & $\begin{array}{c}\text { Non- } \\
\text { evaporated }\end{array}$ & $117.3 \pm 8.8^{\mathrm{bA}}(11.7)$ & $9.6 \pm 4.3^{\text {ав }}$ & $66.4 \pm 7.7 \mathrm{~b}^{\mathrm{A}}$ & $(6.6)$ & $17.8 \pm 7.7^{\mathrm{aB}}$ & $18.3 \pm 1.6^{b}$ \\
\hline & Evaporated & $135.3 \pm 56.9^{\mathrm{abA}}(13.5)$ & $8.6 \pm 4.3^{\mathrm{aB}}$ & $78.7 \pm 35.2^{\mathrm{a}}$ & $\mathrm{AA}(7.8)$ & $20.3 \pm 7.9^{\mathrm{aB}}$ & $21.3 \pm 9.2^{b}$ \\
\hline \multirow[t]{2}{*}{$\mathrm{OP}$} & $\begin{array}{c}\text { Non- } \\
\text { evaporated }\end{array}$ & $133.0 \pm 18.9^{\mathrm{abA}}(13.3)$ & $5.5 \pm 7.9^{\mathrm{aB}}$ & $82.4 \pm 6.9^{\mathrm{ab} /}$ & $(8.2)$ & $20.1 \pm 16.5^{\mathrm{aB}}$ & $21.5 \pm 2.5^{b}$ \\
\hline & Evaporated & $154.6 \pm 5.4^{\mathrm{aA}}(15.4)$ & $5.9 \pm 8.6^{\mathrm{aB}}$ & $91.9 \pm 5.8^{\mathrm{aA}}$ & $(9.1)$ & $17.5 \pm 6.6^{\mathrm{aB}}$ & $24.5 \pm 0.9^{b}$ \\
\hline \multirow[t]{2}{*}{ SBU } & $\begin{array}{c}\text { Non- } \\
\text { evaporated }\end{array}$ & $113.3 \pm 10.3^{\mathrm{bA}}(11.3)$ & $5.3 \pm 6.8^{\mathrm{aB}}$ & $71.9 \pm 13.9$ & $\mathrm{BA}(7.1)$ & $18.4 \pm 11.9^{\mathrm{aB}}$ & $18.4 \pm 2.2^{\mathrm{b}}$ \\
\hline & Evaporated & $113.2 \pm 4.6^{\mathrm{bA}}(11.3)$ & $8.9 \pm 1.8^{\mathrm{aB}}$ & $66.5 \pm 6.5^{\mathrm{b} f}$ & (6.6) & $19.6 \pm 7.4^{\mathrm{aB}}$ & $17.9 \pm 0.9^{b}$ \\
\hline
\end{tabular}

Values are Mean $\pm S D(n=12) \mu \mathrm{g} / \mathrm{mm}^{3}$

Different upper case letters for rows and lower case letters for columns: significant $(p<0.05)$

Water sorption is given in absolute terms $\left(\mathrm{g} / \mathrm{mm}^{3}\right)$ and in relative terms (\%) to provide comparisons to literature values which include both expressions

C $57.1 \mu \mathrm{g} / \mathrm{mm}^{3}=0.5710 \mathrm{mg} / \mathrm{mm}^{3} \times 1005.71 \mathrm{mg} / 100 \mathrm{~mm}^{3}=5.7 \%$ [Malacarne, et al. ${ }^{12}$ (2006)]

D Net water uptake is the sum of water sorption and solubility (\%) 


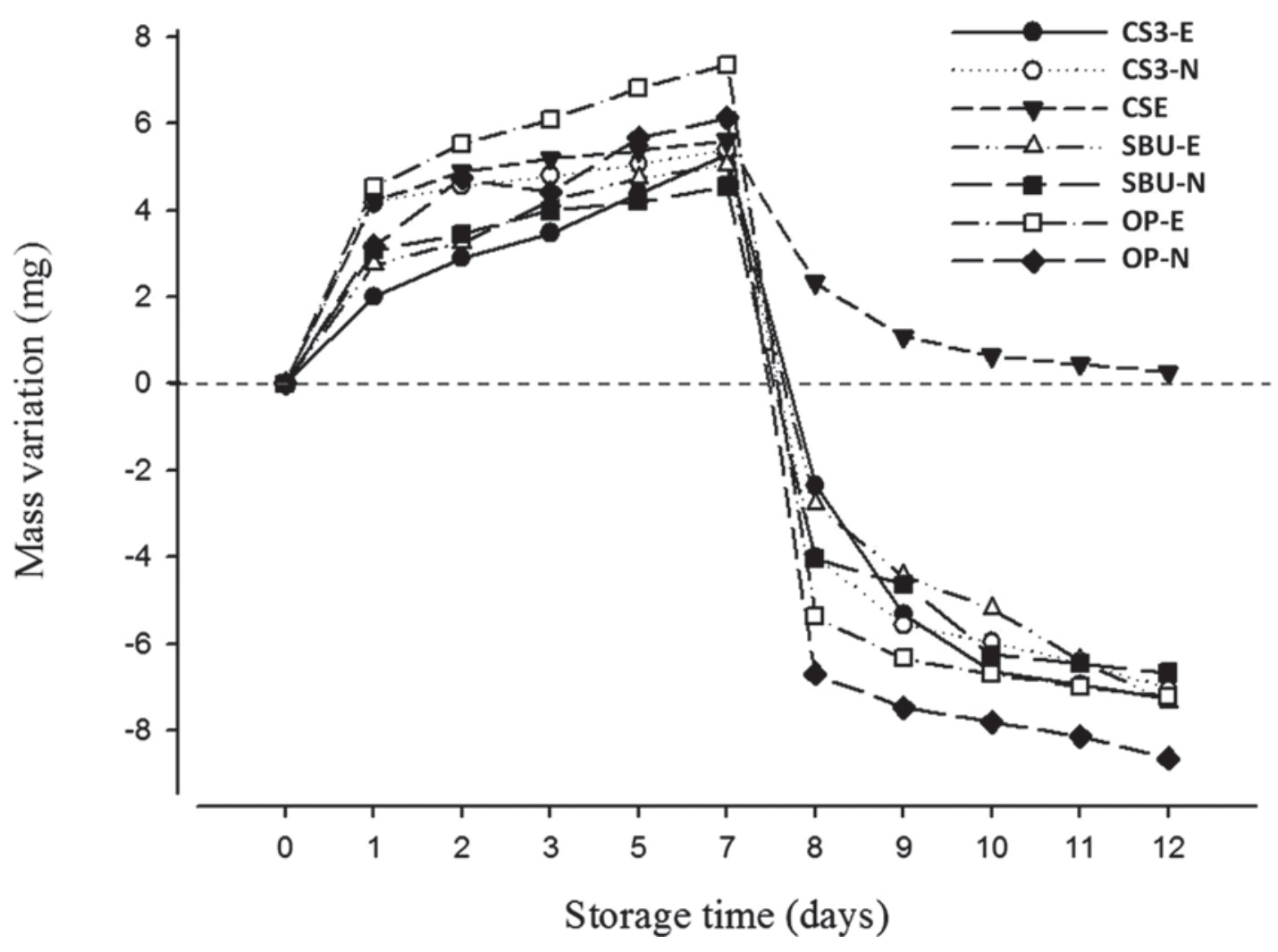

Figure 2- Changes in mass of the adhesive systems tested in the water sorption/solubility test. Symbols represent mean values ( $\mathrm{N}=12)$. Standard deviation values were not indicated. OP - Optibond Solo Plus, CS3 - Clearfil $\mathrm{S}^{3}$ Bond, and SBU Scotchbond Universal Adhesive as a function of the solvent evaporation (non-evaporated - N) or evaporated solvents - E)
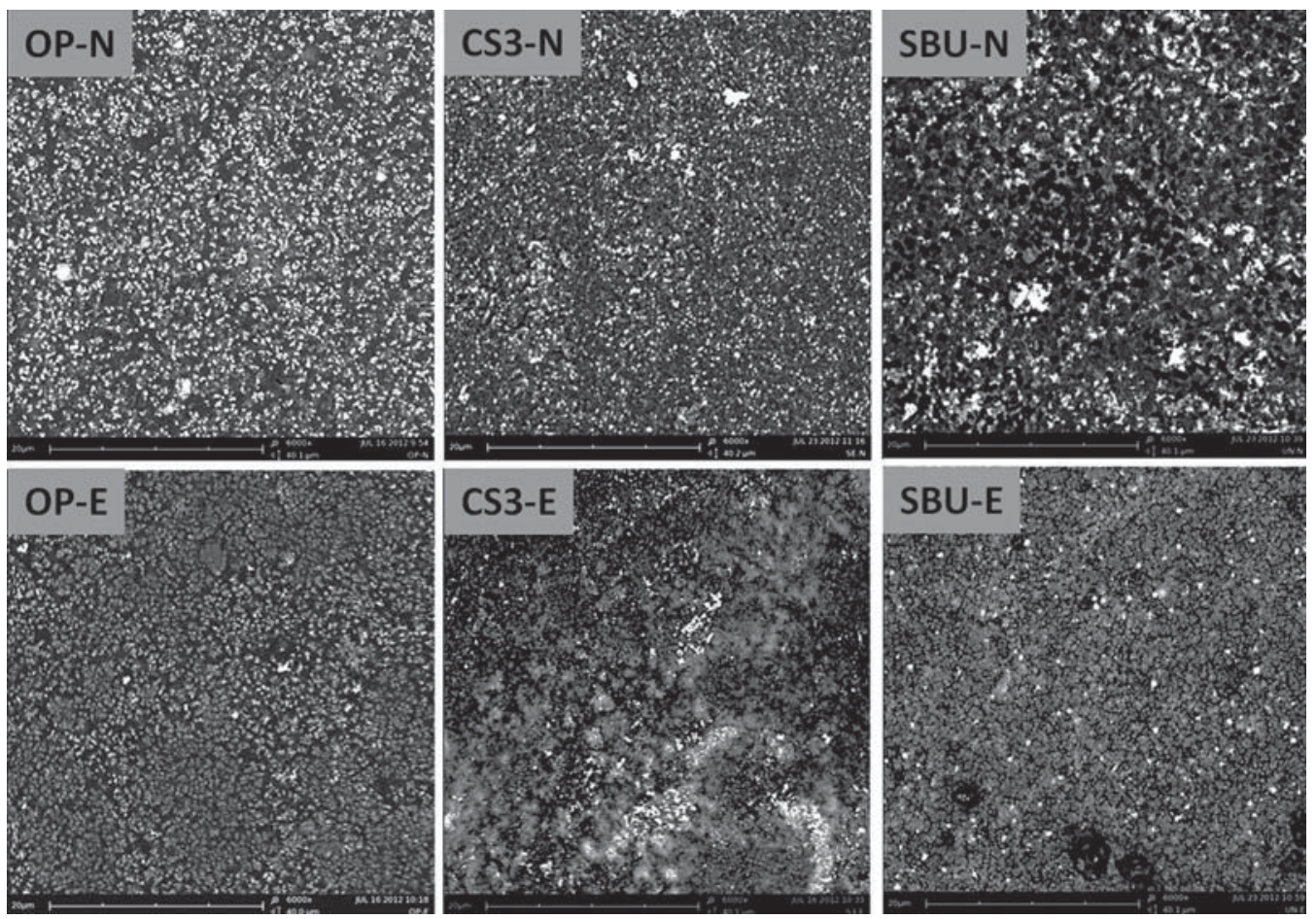

Figure 3- Nanoleakage of the adhesives Optibond Solo Plus (OP), Clearfil $\mathrm{S}^{3}$ Bond (CS3), and Scotchbond Universal Adhesive (SBU) with the solvent non-evaporated $(\mathrm{N})$ or evaporated $(\mathrm{E})$ after 7 days of water storage. The more intense silver uptake is evident for the non-evaporated groups 


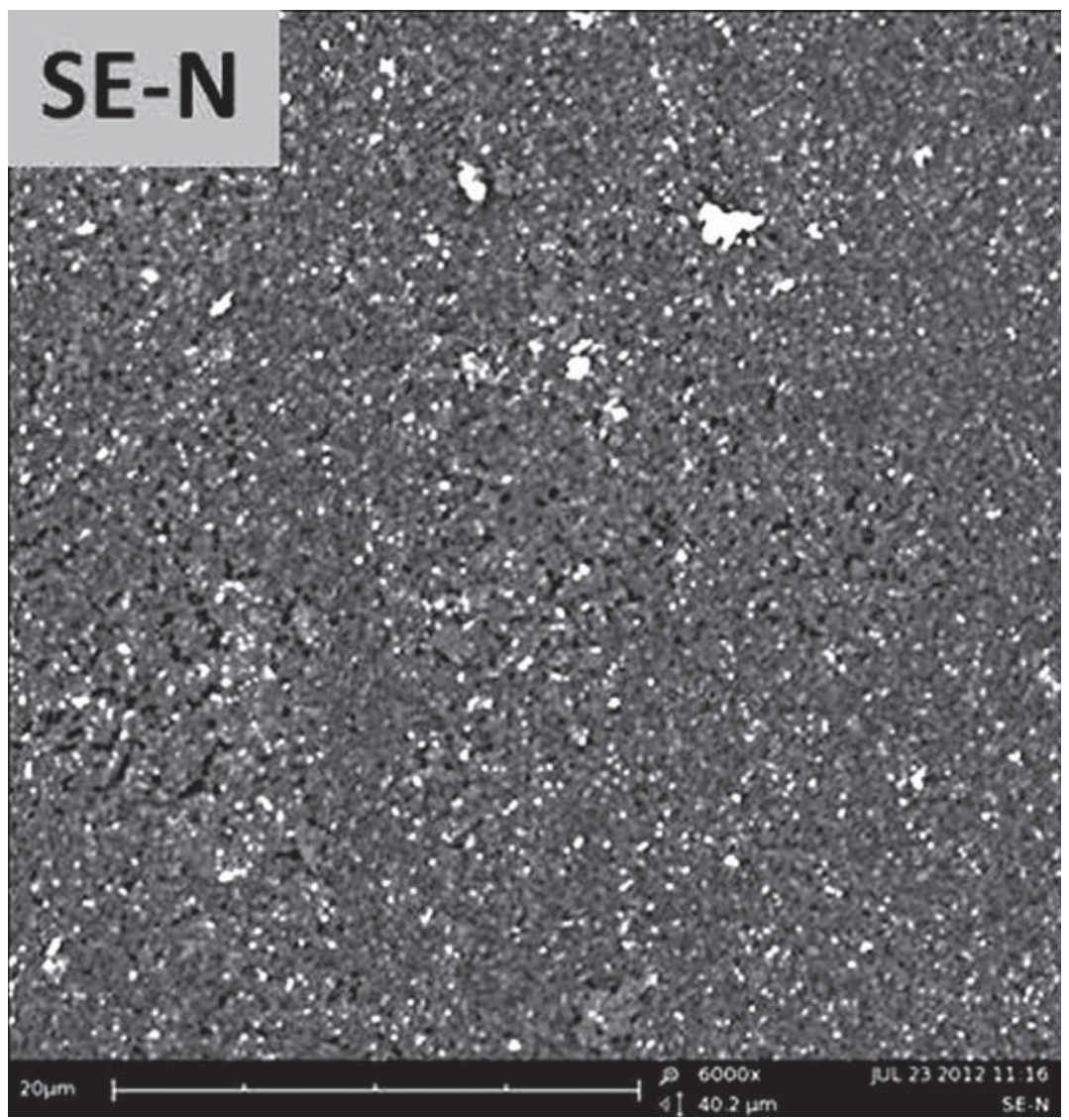

Figure 4- Nanoleakage of Clearfil SE Bond (CSE) after 7 days of water storage

Representative SEM micrographs of the adhesives after 7 days of storage in water are presented in Figure 3. The solvated adhesives showed similar nanoleakage patterns (moderate silver uptake Type I) after evaporation, whereas a more intense nanoleakage was noted for the non-evaporated ones (severe silver uptake - Type II) (Figure 3). The nonsolvated adhesive CSE presented a nanoleakage pattern (Type I) similar to that observed for the solvated adhesives when the solvents were evaporated (Figure 4). The adhesive SBU showed higher silver uptake when the evaporation was not performed in comparison with that observed for both OP and CS3.

\section{DISCUSSION}

The first hypothesis that the solvent evaporation procedure would reduce the net water uptake was not accepted. In the present study, the tested adhesives showed different amounts of net water uptake, which was not dependent on the evaporation of the solvent. Although the WS and SL means varied among the experimental groups, no significant difference was observed among the groups, irrespective of the storage media. In a previous study ${ }^{1}$, higher solvent eliminations were only observed when the drying air temperature was raised for adhesives in comparison to non- evaporated groups.

The net water uptake, which represents the sum of water sorption/solubility parameters ${ }^{25}$, has been claimed to reliably estimate the capacity of polymers to absorb water ${ }^{23}$. It has been pointed out that simply evaluating the increase in mass of the specimens stored in water is not the correct way of assessing their water sorption ${ }^{12}$. Hydrophilic methacrylate polymers exhibit a spatial heterogeneity in which some parts are densely cross-linked and some parts are loosely crosslinked, representing a variable density of crosslinks ${ }^{12}$. In this way, there may be a dynamic process when resin-based materials are stored in water that interferes in the mass of these materials ${ }^{23}$. Thus, there may be a simultaneous infiltration of water into the materials while unreacted monomers and low-molecular-weight polymers may be leached out $^{21-22}$. In this way, the mass variation as a result of both an increase in mass due to water penetration and a decrease in mass as a function of the elution of low-molecular-weight material has been advocated ${ }^{12}$. This association was claimed to represent the net water uptake ${ }^{11}$.

The storage of the specimens in mineral oil exhibited significantly lower WS and SL when compared to that of the specimens stored in water. When the WS and SL were compared among the adhesive groups, no significance was observed 
among the groups, irrespective of the evaporation of the solvents. In addition, no significance was observed for the non-solvated adhesive (CSE Bond) concerning the means exhibited by the experimental groups. Thus, the second hypothesis, which anticipated that there would be no difference in the WS and SL parameters when the immersion media were compared, was rejected. It has been demonstrated that when specimens were immersed in oil, no water to challenge the interfaces existed, which decreased the silver impregnation to a minimum amount ${ }^{18}$. It has been pointed out that the storage in oil might have removed residual water from the resin-dentin interfaces. Even though water is barely solubilized by the mineral oil, the oil can dehydrate the adhesive specimens making them stiffer. In addition, in the absence of water, less silver uptake may occur ${ }^{18}$.

Many factors may affect the water sorption and solubility of contemporary dentin-bonding systems. Polymers absorb moisture in a humid atmosphere or when immersed in water. Moisture diffuses into polymers at different degrees depending on a number of molecular and microstructural aspects: (1) polarity of the molecular structure, presence of chemical groups capable of forming hydrogen bonds with water; (2) degree of crosslinking; (3) presence of residual monomers; and (4) crystallinity of the polymer (well-defined crystallites are inaccessible to water $)^{15}$. According to these factors, the mechanism of water diffusion can be summarized in two main theories: (1) free-volume theory, according to which water diffuses through voids within the polymer, and (2) interaction theory, according to which water binds to specific ionic groups of the polymer chain ${ }^{12}$. In this case, water diffusion occurs according to the water-affinity of these groups ${ }^{12,14}$. It was reported that the amount of water sorption and solubility of adhesive polymers increased proportionally to their HEMA concentrations ${ }^{9,16}$. In another study, it was pointed out that monomers are heteroatom polymers, having carbon and oxygen or nitrogen in their backbones and that the presence of certain groups, such as ester, urethane, and ether linkages, as well as hydroxyl groups are hydrolytically susceptible ${ }^{8}$. Despite their relative hydrophilicity, the resultant polymer formed may absorb water to a potentially damaging extent ${ }^{8}$.

Moreover, the solvents are probably another factor regarding the extent of water sorption and solubility. Solvents also contribute to an exacerbation of the hydrophilicity of the adhesives ${ }^{12}$. Additionally, another study ${ }^{7}$ demonstrated differences in the water sorption of commercial formulations of bonding agents, which indicated the presence of residual solvents as a condition to exacerbate water sorption. This exerts a negative influence on the bonding longevity, as varied resinous monomers are combined into solvents as neat acetone, ethanol, water, or their combination ${ }^{17,19}$. A previous study also pointed out the fact that the solvent acetone spontaneously evaporates, as its vapor pressure and ebullition temperature are lower than those of other solvent, which results in higher evaporation rate ${ }^{9}$.

In the present study, the highest water sorption and solubility means were obtained for solvated adhesive formulations compared to those of CSE Bond. Negative values were obtained for water solubility for CSE. This may indicate that the adhesive is more suitable to water sorption, which could mask its real solubility. The results demonstrate that the kinetics of water uptake was also material-dependent. Thus, the data obtained with the non-solvated adhesive as a parameter for the relationship between water sorption and hydrophilicity suggests that CS3, OP, and SBU present, in fact, more hydrophilic behavior than CSE. This is probably due to the solvents present in the composition ${ }^{12}$, i.e., the rate of water uptake was lower for the less-hydrophilic adhesive CSE.

The solvated adhesives investigated in the present study utilize the same types of solvents (water and ethanol), which could explain the similar values for net water uptake. Although the air-drying had no effect on the net water uptake of the adhesives, residual solvent remaining after the air-drying might produce defects within the polymerized adhesive, which was filled with water molecules during storage. The interpretation of the results was improved by the nanoleakage investigation, in which the silver uptake was significantly increased when the solvated adhesives were not submitted to solvent evaporation prior to storage (Figures 3 and 4), rejecting hypothesis III. Thus, it is possible that the water sorption and silver uptake in the adhesive specimens were possibly due mainly to porous formation of the polymerized adhesive caused by the presence of residual solvents.

Another important consideration is that all the adhesives tested in the present study contain HEMA, and residual co-monomer mixtures may be attracted by poly-HEMA and/or polymers, leading to an increase in the free volume of polymerized adhesive. This promotes an increase in the water sorption into the polymerized matrix, even after airdrying the adhesive prior to immersion in water ${ }^{11}$. This also helps to explain the nanoleakage for the groups in which the solvent was evaporated, as well as for the non-solvated CSE (Figures 3 and 4 ). Thus, water molecules diffuse through the porous structure in the adhesive specimens without a mutual relationship with the polar sites of the material $^{8}$. 


\section{CONCLUSION}

Within the limitations of the present study, it can be concluded that although the solvent evaporation related to the adhesive protocol has no influence on the net water uptake of the adhesives evaluated, the nanoleakage was significantly affected for the non-evaporated ones.

\section{ACKNOWLEDGEMENTS}

The authors are grateful to Prof. Dr. Francisco André Ossamu Tanaka from NAP/MEPA (Support Research Nucleus / Electron Microscopy Applied to Agriculture) - ESALQ/USP - Piracicaba for the support in the SEM analysis.

\section{REFERENCES}

1- Bail M, Malacarne-Zanon J, Silva SM, Anauate-Netto A, Nascimento FD, Amore R, et al. Effect of air-drying on the solvent evaporation, degree of conversion and water sorption/ solubility of dental adhesive models. J Mater Sci Mater Med. 2012;23(3):629-38.

2- Breschi L, Cadenaro M, Antoniolli F, Sauro S, Biasotto M, Prati $C$, et al. Polymerization kinetics of dental adhesives cured with LED: correlation between extent of conversion and permeability. Dent Mater. 2007;23(9):1066-72.

3- Carvalho RM, Manso AP, Geraldeli S, Tay FR, Pashley DH. Durability of bonds and clinical success of adhesive restorations. Dent Mater. 2012;28(1):72-86.

4- D'Alpino PH, Svizero NR, Pereira JC, Rueggeberg FA, Carvalho RM, Pashley $\mathrm{DH}$. Influence of light-curing sources on polymerization reaction kinetics of a restorative system. Am J Dent. 2007;20(1):46-52.

5- Dhanpal P, Yiu CK, King NM, Tay FR, Hiraishi N. Effect of temperature on water sorption and solubility of dental adhesive resins. J Dent. 2009;37(2):122-32.

6- Dickens $\mathrm{SH}$, Cho $\mathrm{BH}$. Interpretation of bond failure through conversion and residual solvent measurements and Weibull analyses of flexural and microtensile bond strengths of bonding agents. Dent Mater. 2005;21(4):354-64.

7- Fabre HS, Fabre S, Cefaly DF, Oliveira Carrilho MR, Garcia FC, Wang L. Water sorption and solubility of dentin bonding agents light-cured with different light sources. J Dent. 2007;35(3):253-8.
8- Ferracane JL. Hygroscopic and hydrolytic effects in dental polymer networks. Dent Mater. 2006;22(3):211-22.

9- Garcia FC, Wang L, Pereira LC, Andrade e Silva SM, Júnior LM, Carrilho MR. Influences of surface and solvent on retention of hHEMA/mixture components after evaporation. J Dent. 2010;38(1):44-9.

10- Ikeda T, De Munck J, Shirai K, Hikita K, Inoue S, Sano H, et al. Effect of air-drying and solvent evaporation on the strength of HEMA-rich versus HEMA-free one-step adhesives. Dent Mater. 2008;24(10):1316-23.

11- Ito S, Hoshino T, Iijima M, Tsukamoto N, Pashley DH, Saito T. Water sorption/solubility of self-etching dentin bonding agents. Dent Mater. 2010;26(7):617-26.

12- Malacarne J, Carvalho RM, Goes MF, Svizero N, Pashley DH, Tay FR, et al. Water sorption/solubility of dental adhesive resins. Dent Mater. 2006;22(10):973-80.

13- Moraes LG, Rocha RS, Menegazzo LM, Araújo EB, Yukimito $\mathrm{K}$, Moraes JC. Infrared spectroscopy: a tool for determination of the degree of conversion in dental composites. J Appl Oral Sci. 2008;16(2):145-9.

14- Mortier E, Gerdolle DA, Jacquot B, Panighi MM. Importance of water sorption and solubility studies for couple bonding agente - resin-based filling material. Oper Dent. 2004;29(6):669-76. 15- Nishitani Y, Yoshiyama M, Donnelly AM, Agee KA, Sword J, Tay $F R$, et al. Effects of resin hydrophilicity on dentin bond strength. J Dent Res. 2006;85(11):1016-21.

16- Nishitani Y, Yoshiyama M, Hosaka K, Tagami J, Donnelly A, Carrilho $M$, et al. Use of Hoy's solubility parameters to predict water sorption/solubility of experimental primers and adhesives. Eur J Oral Sci. 2007;115(1):81-6.

17- Perdigão J, Frankenberger R. Effect of solvent and rewetting time on dentin adhesion. Quintessence Int. 2001;32(5):385-90. 18- Reis AF, Giannini M, Pereira PN. Long-term TEM analysis of the nanoleakage patterns in resin-dentin interfaces produced by different bonding strategies. Dent Mater. 2007;23(9):1164-72. 19- Reis AF, Oliveira MT, Giannini M, De Goes MF, Rueggeberg FA. The effect of organic solvents on one-bottle adhesives' bond strength to enamel and dentin. Oper Dent. 2003;28(6):700-6. 20- Sano H, Yoshikawa T, Pereira PN, Kanemura N, Morigami M, Tagami J, et al. Long-term durability of dentin bonds made with a self-etching primer, in vivo. J Dent Res. 1999;78(4):906-11.

21- Sideridou I, Achilias DS, Spyroudi C, Karabela M. Water sorption characteristics of light-cured dental resins and composites based on Bis-EMA/PCDMA. Biomaterials. 2004;25(2):367-76. 22- Sideridou I, Tserki V, Papanastasiou G. Study of water sorption, solubility and modulus of elasticity of light-cured dimethacrylatebased dental resins. Biomaterials. 2003;24(4):655-65. 